\title{
Analysis of force and impulse of a hydrophobic rod rotating into water
}

\author{
Sun Gi Park, Min Chul Lee, Hyeong Jun Tak and Jong Soo Ko*
}

\begin{abstract}
This paper investigates the change of the relative magnitudes of force and impulse components generated by the rotational motion of a hydrophobic carbon rod moving into water from the water surface, by varying the rod length. Whereas added mass force and drag force were dominant regardless of the rod length, buoyancy force and surface tension force were relatively small. For relatively short rod lengths $(2,4,6 \mathrm{~cm})$, drag forces were larger than added mass forces in the beginning of the rotational motion; the magnitudes of the two forces, however, reversed during the rotation. It was found that the reversals of magnitudes of the two forces occurred when the velocity at the end of the rod was $0.5 \mathrm{~m} / \mathrm{s}$. On the other hand, for a long rod length of $8 \mathrm{~cm}$, added mass force was higher than drag force throughout the rod motion. The change of impulse by varying the rod length showed a similar tendency to that of force. Added mass impulse and drag impulse were also considerably larger than buoyancy impulse and surface tension impulse. Furthermore, drag impulse was larger than added mass impulse for short rod lengths, and the magnitudes of the two impulses reversed for long rod lengths. Compared to the shorter rod, the larger rod has a relatively low momentary force, but its force has much larger duration time, resulting in an increase of impulse.
\end{abstract}

\section{Introduction}

All living things have unique moving methods that have been developed through evolution. Creatures that walk or run on the ground use frictional or reaction forces. The surface of the earth is solid and hard and thus, sufficient frictional and reaction forces can be provided. In contrast, because the surface of water is easily deformed by external forces, the frictional and reaction forces from the water surface are much smaller than those from a solid surface. Like animals that move on solid ground, those moving on a water surface also can walk, run, and jump on it. Animals that are relatively large, however, cannot move on the water surface due to their heavy weight. The Basilisk lizard, among relatively large reptiles, can run on water by slapping the water with its back feet. Fringes between its toes create a large surface area. When the foot slaps the water, the large foot surface makes a large hole in the water, and the generated buoyancy pushes up the lizard. It has been measured that

\footnotetext{
*Correspondence: mems@pusan.ac.kr

Graduate School of Mechanical Engineering, Pusan National University,

Busandaehak-ro 63beon-gil, Geumjeong-gu, Busan 46241, Republic of Korea
}

this motion produces $110-225 \%$ of the force needed to support the lizard's weight [1]. However, while the Basilisk lizard can move on water through his quick slapping motion, it cannot stand on the water surface due to its heavy weight.

The water strider, which is much smaller and lighter than Basilisk lizard, is representative of insects that live on water. The water strider's legs are superhydrophobic; the surface tension force created by the legs can support up to 15 times its weight [2]. Therefore, water striders can stand, move, and jump on the surface of water. Water striders jump in order to overcome the limitation of movement and escape predatory threats [3]. Many researchers have recently developed water strider robots that mimic the shape and movement of natural water striders [4-7]. There are two major trends in this research: development of an actuation mechanism to increase the moving speed [4] and increase of the surface tension force by surface modification of the legs [5-7]. However, previous studies have mostly focused on forward movement on the water surface; little research has been carried out on jumping. Shin et al. conducted research that represents the first step toward a water 
jumping robot that emulates the ability of the water strider and a fishing spider to jump on the water surface $[8,9]$. A shape memory alloy actuator was used for the slapping motion of the legs. The robot jumped by pushing the water surface with rapidly released legs that were initially bent. They successfully measured the jumping height according to the bending angle of the legs, and showed the water jumping efficiency versus the bending angle. The water jumping efficiency was defined as the ratio of the maximum jumping height on the water to that on the ground.

However, analysis of each force element and each impulse element generated by each force while the robot legs push the water was not conducted. Impulse is the physical quantity that has a direct effect on the jumping height of a jumping object [10]. We aim to analyze each force element and each impulse element when hydrophobic rods have rotational motion with high speed moving into water from the water surface. In addition, we investigate the change of the impulse generated from each force by varying the rod length. In order to analyze the force and impulse acting on the rods in the water, we constrained the rotational motion of the rods in the water in this study.

\section{Theory: force and impulse generated by the movement of the rod on the water}

When a hydrophobic rod strikes a water surface, as shown in Fig. 1a, the instantaneous force (F) acting on the rod can be divided into two main categories: the force acting area $(S)$ of the rod in direct contact with the water and the force acting on the contact line $(C)$ of the rod
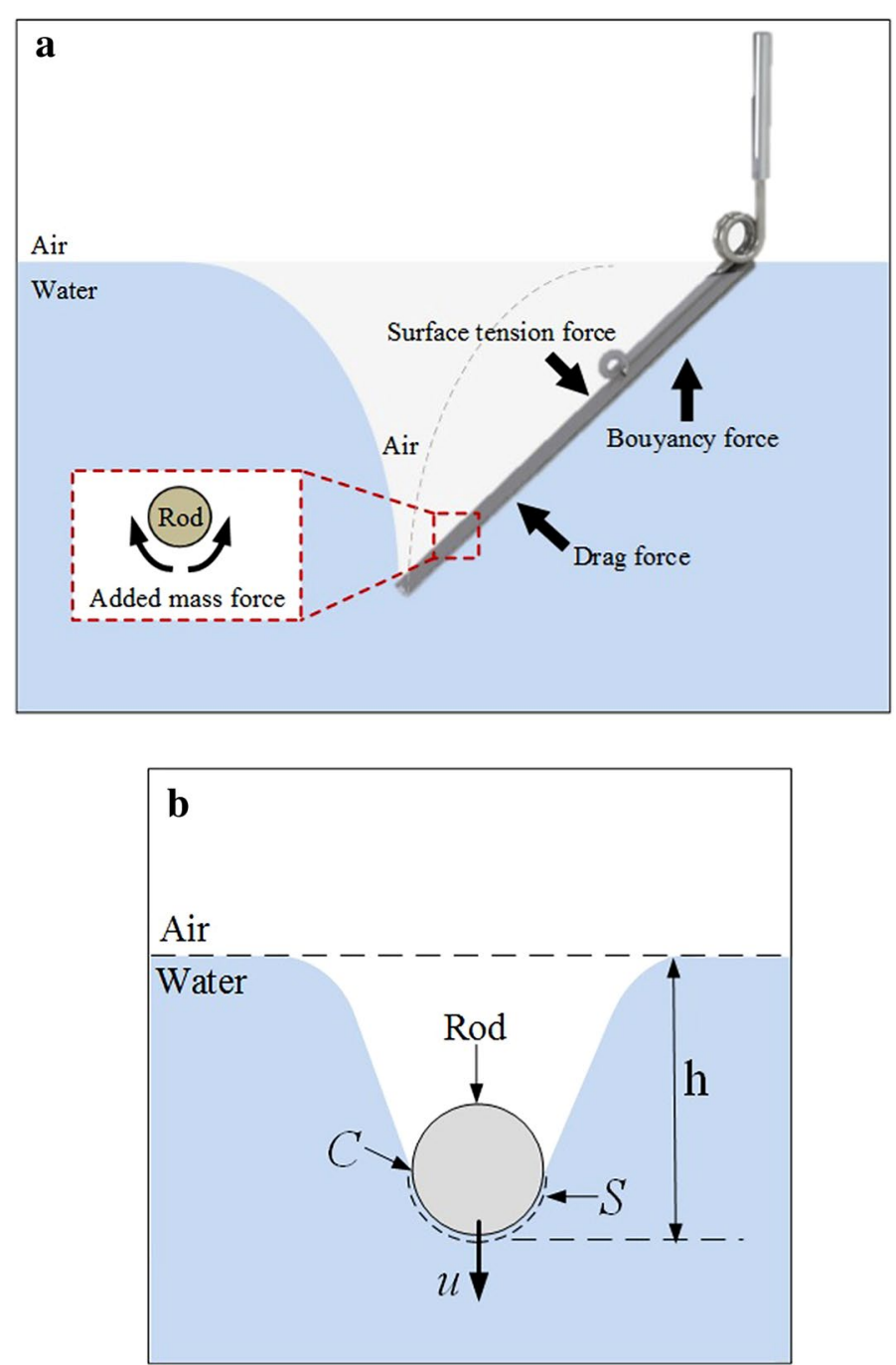

Fig. 1 Four major reaction forces acting on a rotating carbon rod (a) and its cross section (b) 
with the water surface. If the weight of the rod is ignored, the instantaneous force can be written as the summation of these two forces:

$$
\mathbf{F}=\int_{S} \mathbf{T} \cdot \mathbf{n} d S+\int_{C} \gamma w d L
$$

Here, $\mathbf{T}=-p \mathrm{I}+2 \mu \mathbf{E}$ is the hydrodynamic stress tensor, $p$ the fluid pressure, I the identity matrix, $\mu$ the viscosity, and $\mathbf{E}$ the rate of strain tensor defined as $\left[\nabla u+(\nabla u)^{\mathbf{T}}\right] / 2$. Here, $u$ represents the velocity of the rod. $p$ can be expressed by the time-dependent Bernoulli equation [11-13]:

$$
\frac{\partial \varphi}{\partial \mathrm{t}}+\frac{u^{2}}{2}+\frac{\mathrm{p}}{\rho}+g h=c
$$

where $\phi$ is the velocity potential and c is a constant. Substituting $p$ obtained from Eq. (2) into the stress tensor $\mathbf{T}$, the force can then be expressed as

$$
\begin{aligned}
\mathbf{F}= & \rho \int_{S} \frac{\partial \varphi}{\partial \mathrm{t}} \mathbf{n} d S+\int_{S} \frac{1}{2} \rho u^{2} \mathbf{n} d S+\int_{S} \rho g h \mathbf{n} d S \\
& +2 \mu \int_{S} \mathrm{E} \cdot \mathbf{n} d S+\int_{C} \gamma w d L
\end{aligned}
$$

Each term of the right hand side represents the added mass force, drag force, buoyancy force, viscous force, and surface tension force. The viscous force is negligible when the bulk Reynolds number is large [11-13]. Therefore, impulse, which is defined by the integral of the instantaneous force with respect to time, can be expressed as

$$
\begin{aligned}
\mathbf{J}= & \int_{t}\left\{\rho \int_{S} \frac{\partial \varphi}{\partial t} \mathbf{n} d S+\int_{S} \frac{1}{2} \rho u^{2} \mathbf{n} d S\right. \\
& \left.+\int_{S} \rho g \mathrm{~h} \mathbf{n} d S+\int_{C} \gamma w d L\right\} \mathrm{dt}
\end{aligned}
$$

Added mass impulse, drag impulse, buoyancy impulse, and surface tension impulse can be obtained from Eq. (4). Because the forces acting vertically from the water surface have a direct effect on jumping, the vertical components were extracted from the forces and thus the vertical impulse components were considered. It is assumed that the rod comes into contact with the water at its lower half while rotating in this study.

\section{Design and fabrication}

\section{Working mechanism and design}

Figure 2a, b show a carbon rod that is bonded with a torsion spring. The other side of the torsion spring is inserted into an aluminum tube and bonded. A string, which connects the aluminum tube and the carbon rod, folds the carbon rod as shown in Fig. 2a. The carbon rod in this folded initial state is laid parallel on the water surface and the angle between the aluminum tube and the carbon rod is $90^{\circ}$. As soon as the string is severed, as shown in Fig. $2 \mathrm{~b}$, the carbon rod begins to rotate into the water. The aluminum tube does not move during the rotation of the carbon rod.

As shown in Fig. 2c, the center part of the torsion spring is wound twice with a center diameter of $2.5 \mathrm{~mm}$. While the left end of the torsion spring is wound once with a diameter of $1 \mathrm{~mm}$, the right end is straight. The 4-mm-long right side is inserted into a hollow aluminum tube and bonded with epoxy. The 11-mm-long left side is bonded with a carbon rod and the ring at the left end is connected with a string, the length of which controls the bending angle of the torsion spring. The angle between the left and right sides is designed to be $150^{\circ}$. The surfaces of the carbon rods are hydrophobically modified as shown in Fig. 2d. In order to investigate the change of the forces and impulses according to the rod length, four different rod lengths were designed: 2, 4, 6 and $8 \mathrm{~cm}$.

\section{Fabrication}

Spring steel with $0.35 \mathrm{~mm}$ diameter was wound twice with a center diameter of $2.5 \mathrm{~mm}$. The spring constant of the fabricated torsion springs was $0.0584 \mathrm{mN} \mathrm{m} / \mathrm{deg}$ (measurement system: PRO-TS-50-E-220, K\&K Trading, Korea). The straight-type right part of the fabricated spring was inserted into an aluminum tube of which the inner diameter was $2.5 \mathrm{~mm}$, and then bonded using epoxy (Alteco F-05, Alpha Techno, Japan). The ringtype left part was adhered on the carbon tube (Midwest, USA) using the same epoxy. Inner and outer diameters of the hollow-type carbon rod were $0.3 \mathrm{~mm}$ and $0.7 \mathrm{~mm}$, respectively. The carbon rod was coated with a sol-gel solution (SS20, NanoM, Korea). The sol-gel solution based on fluoroalkyl silane and tetraorthosilicate contains silica nanoparticles with $15 \mathrm{~nm}$ diameter to lend a hydrophobic nature. Figure $2 \mathrm{~b}$ shows a fabricated sample with a 6-cm-long carbon rod. The angle between the carbon rod and the aluminum fixture is decreased by controlling the length of the Teflon string, which is $0.4 \mathrm{~mm}$ in diameter (Nfive, Xmesis, Japan) and connects the connection ring of the torsion spring and the aluminum fixture, as shown in Fig. 2a. While the carbon rod makes an angle of $90^{\circ}$ with the aluminum fixture in the triggered state (Fig. 2a), it makes an angle of $150^{\circ}$ in the released state (Fig. 2b). Figure $2 \mathrm{~d}$ shows that the combination of the rough surface morphology of the nanoparticles and the hydrophobicity of the sol-gel solution modifies the 


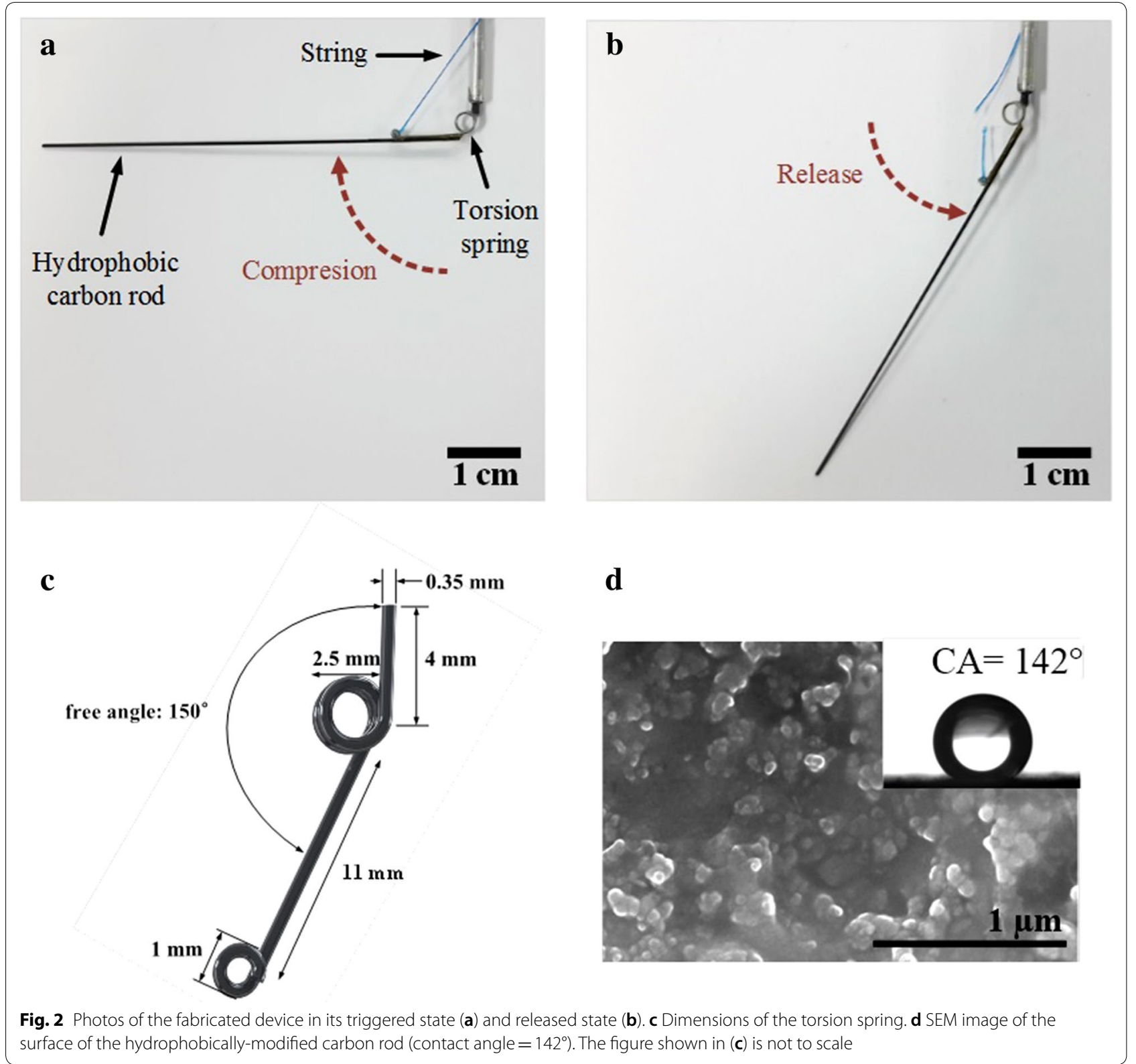

surface of the rod to be strongly hydrophobic (with a measured contact angle of $142^{\circ}$ ).

\section{Measurements and discussion}

\section{Measurement methods and results}

The triggered carbon rod starts to rotate as soon as the Teflon string is cut. The center of the string is cut using a razor (ST-300, Dorco, Korea) that is heated until it turns red to minimize external force acting on the string during cutting. Rotational motion of the carbon rod in the water is captured using a high-speed CCD (charge coupled device) camera (Phantom, Vision research, USA). While a carbon rod makes an angle of $90^{\circ}$ with the aluminum fixture in the triggered state (Figs. 2a, 3a), it makes an angle of $150^{\circ}$ with the aluminum fixture when the rotational motion of the carbon rod is finished (Figs. 2b, 3c). A frame rate of $2550 \mathrm{fps}$ (exposure time: $400 \mu \mathrm{s}, 392 \mu \mathrm{s}$ ) has been set for capturing images. The Ph630 program (Phantom, Vision research, USA) was used to measure the angle between the water surface and the carbon rod from the captured images. The carbon rod is on the water surface in the triggered state (Fig. 3a), and consequently the angle between them is zero. Henceforth, the angle of rotation refers to the angle between the carbon rod and 

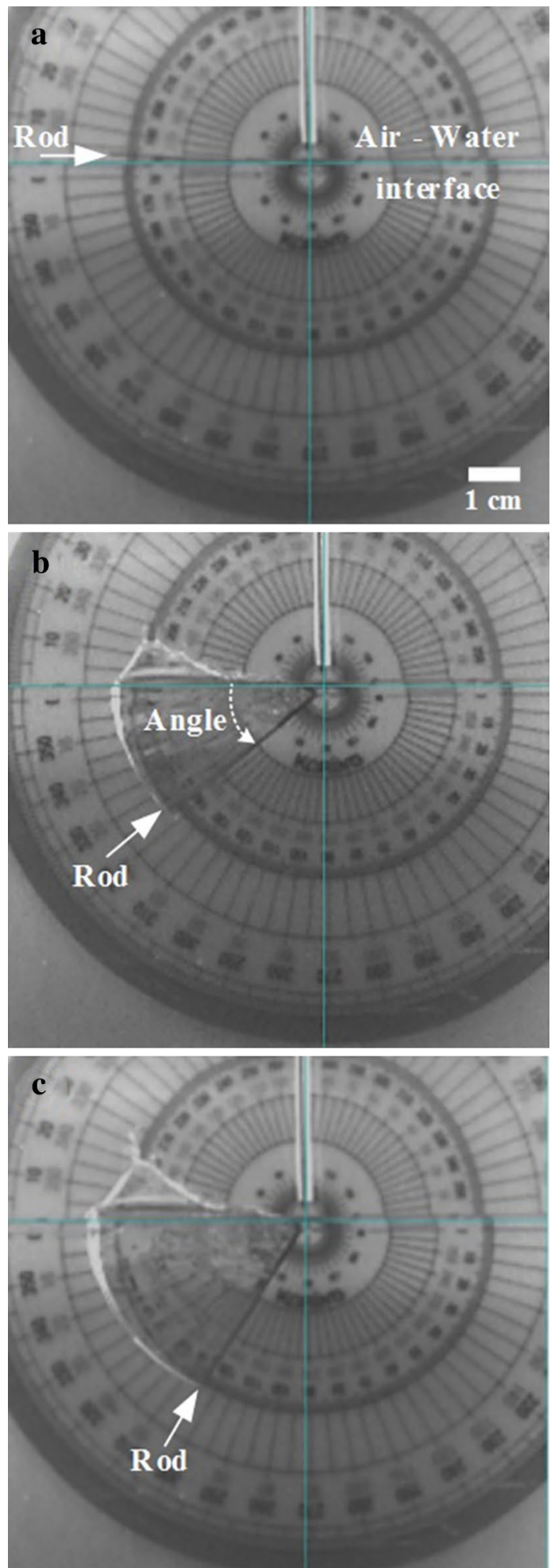

Fig. 3 Time lapse series of images of rotation of a 4-cm-long rod: a $0 \mathrm{~ms}, \mathbf{b} 4 \mathrm{~ms}$, and $\mathbf{c} 8.4 \mathrm{~ms}$ the water surface. Figures $3 \mathrm{a}-\mathrm{c}$ are respective time lapse series of images of rotation of a 4-cm-long carbon rod in the water captured at 0,4 , and $8.4 \mathrm{~ms}$. The measurement was taken five times for each sample and the average values were obtained from the measured data and plotted. The time it takes to complete the rotational motion of the carbon rod increased as the length of the carbon rod increased $(2 \mathrm{~cm}: 2.4 \mathrm{~ms}, 4 \mathrm{~cm}: 8.4 \mathrm{~ms}, 6 \mathrm{~cm}: 20 \mathrm{~ms}, 8 \mathrm{~cm}$ : $40.4 \mathrm{~ms}$ ), as shown in Fig. 4.

In order to obtain forces and impulses acting on a carbon rod, the carbon rod was divided into very small spans of $0.1 \mathrm{~mm}$ through the entire length of the rod. Forces and impulses acting on each span were obtained and they were then integrated through the whole length of the carbon rod. As shown in Fig. 1b, it was assumed that only the lower half of the rod surface contacts the water during the rotational motion of the carbon rod. Because vertical components of forces and impulses to the water surface have a direct effect on jumping, respective vertical components of velocity $(u)$ and acceleration potential $(\Delta \phi / \Delta \mathrm{t})$ obtained from each span $(0.1 \mathrm{~mm})$ were inserted in Eqs. (2) and (3). Therefore, hereafter, all forces and impulses described in this paper are components that are vertical to the water surface.

\section{Force elements}

Figure 5 shows the change of the magnitude of four force elements according to time for each rod length. While added mass force and drag force are dominant regardless of the rod length, buoyancy force and surface tension force are relatively small. This is ascertained that high velocity and acceleration caused by rapid rotation of the carbon rod considerably increased the drag force and the added mass force, respectively.

In the case of the rod lengths of 2, 4, and $6 \mathrm{~cm}$, although drag forces were higher than added mass forces when the rotational motion starts, the magnitudes of the two forces reversed during rotation (Fig. 5a-c). Each arrow mark shown in Fig. $5 \mathrm{a}-\mathrm{c}$ represents the point where the two forces are reversed. The rotational angle where the reversal of magnitudes of the two forces occurred became smaller with greater rod length $\left(2 \mathrm{~cm}: 50.7^{\circ}, 4 \mathrm{~cm}: 29.5^{\circ}\right.$, $6 \mathrm{~cm}: 11.20^{\circ}$ ). In these three rod lengths, it was found that the reversals of magnitudes of the two forces occurred when the velocity at the end span of the rod was $0.5 \mathrm{~m} / \mathrm{s}$. Therefore, drag force is larger than added mass force when the velocity of the end span is higher than $0.5 \mathrm{~m} / \mathrm{s}$, but vice versa when it is lower than $0.5 \mathrm{~m} / \mathrm{s}$. On the other hand, added mass force is higher than drag force from start to finish for the rod length of $8 \mathrm{~cm}$. 

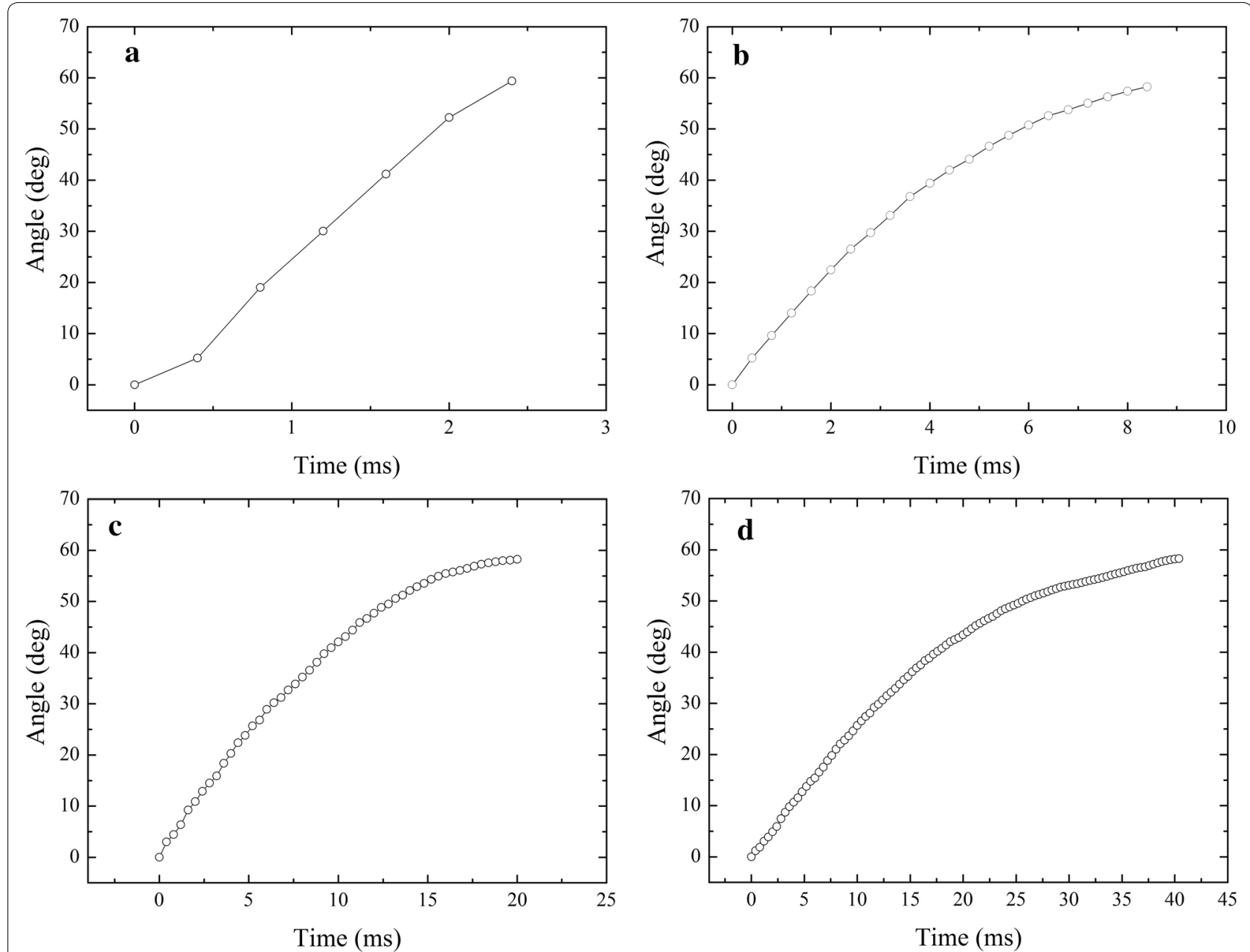

Fig. 4 The change of the rotating angle of each rod according to time: a rod with $2 \mathrm{~cm}$ length, b $4 \mathrm{~cm}, \mathbf{c} 6 \mathrm{~cm}$, and $\mathbf{d} 8 \mathrm{~cm}$

Since the rotational speed is relatively high at the early stage of motion, drag force having a square term of the velocity $(u)$ is larger than added mass force having a linear term of the acceleration $(\Delta \phi / \Delta t)$. When the rotational speed decreases, drag force decreased relatively rapidly due to a decrease in the second order of velocity compared to that of added mass force, which linearly decreased by acceleration; reversal of magnitudes of the two forces consequently occurred. The resistance by water became higher with greater rod length. The rotational speed of the carbon rod decreased relatively rapidly, and thus the force reversal phenomenon occurred at a relatively small rotational angle with larger rod length.

\section{Impulse elements}

Figure 6 shows the change of the magnitude of each impulse element by four force elements according to time for each rod length. Similar to force elements, impulses by added mass force and drag force are considerably larger than those by buoyancy and surface tension forces. In the case of the rod lengths of 2 and $4 \mathrm{~cm}$, as shown in Fig. 6a, b, the drag impulse is larger than that by added mass force throughout the motion period. In the case of $6 \mathrm{~cm}$, as shown in Fig. 6c, the drag impulse is larger than that by added mass force in the early stage; the magnitudes of the two impulses reversed, however, in the middle of the rotational motion. In the case of $8 \mathrm{~cm}$, as shown in Fig. 6d, added mass impulse is larger than that by drag force throughout the motion period.

As shown in Fig. 5, a rod of a short length has a relatively larger momentary maximum force compared to that of a larger length; the duration time of the force is, however, much shorter. For instance, while the $4-\mathrm{cm}$ rod shows a maximum resultant force of $0.61 \mathrm{~N}$ at $0.4 \mathrm{~ms}$ $\left(5.21^{\circ}\right)$ (Fig. $5 \mathrm{~b}$ ), the $8-\mathrm{cm}$ rod shows $0.38 \mathrm{~N}$ at $2.8 \mathrm{~ms}$ $\left(7.45^{\circ}\right)$ (Fig. 5d). The momentary maximum resultant force of the 4-cm rod is 1.6 times larger than that of the $8-\mathrm{cm}$ one. For the duration time of the momentary 

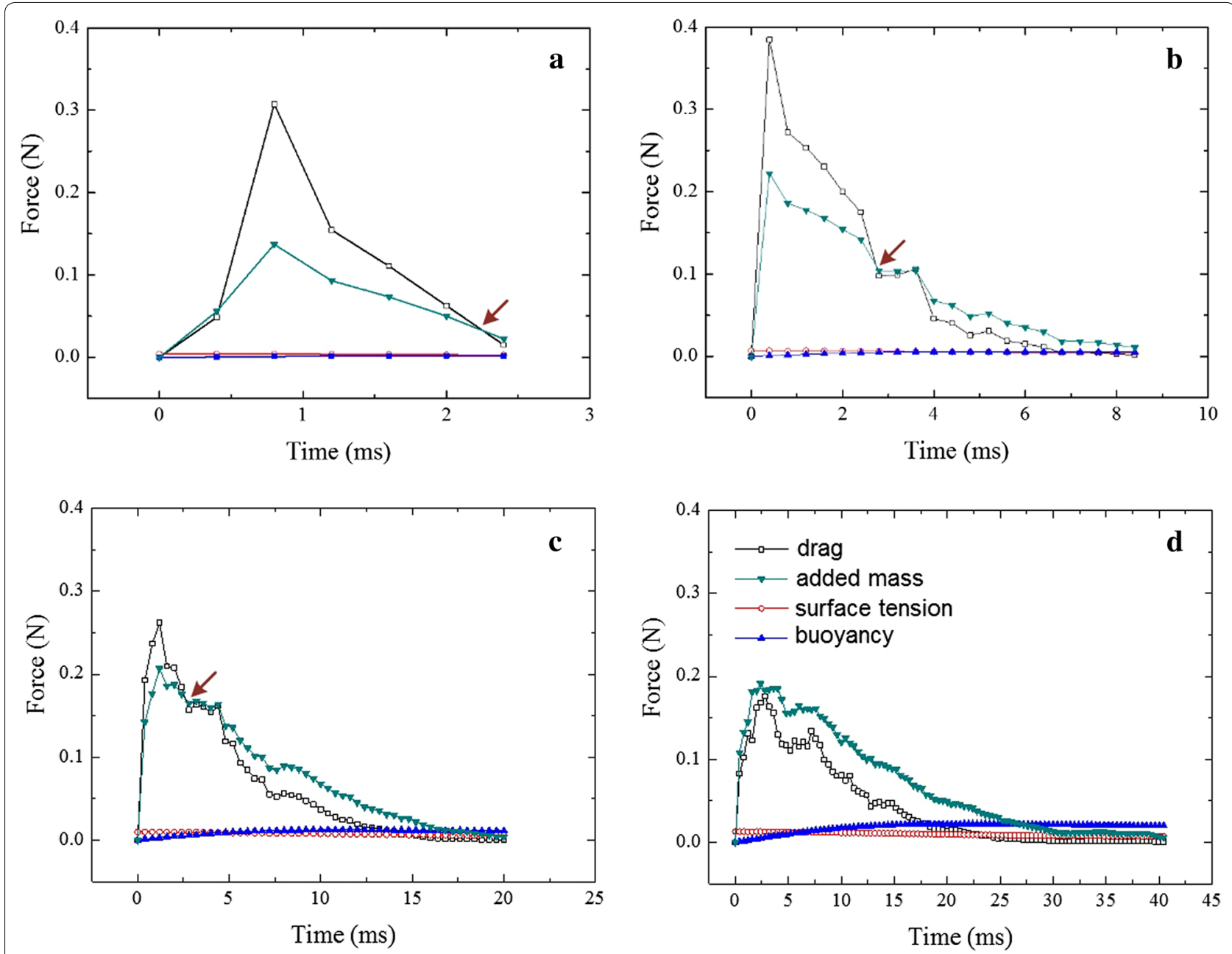

Fig. 5 The change of the magnitude of four force elements according to time for each rod length: $\mathbf{a} 2 \mathrm{~cm}, \mathbf{b} 4 \mathrm{~cm}, \mathbf{c} 6 \mathrm{~cm}$, and $\mathbf{d} 8 \mathrm{~cm}$

resultant force, the 4-cm rod shows a 2.4-ms duration time to maintain a momentary force larger than $0.1 \mathrm{~N}$; the $8-\mathrm{cm}$ rod meanwhile shows an $18.8-\mathrm{ms}$ duration time. The duration time of a momentary resultant force higher than $0.1 \mathrm{~N}$ of the $8-\mathrm{cm}$ rod is 7.8 times longer than that of the 4-cm rod. As a result, the decrement of the maximum momentary resultant force depending on an increase of a rod length is not considerable, but the increment of the duration time of the force is significant; thus the longer the rod length, the higher the impulse. It is expected that, because the jumping height of a jumping object is directly influenced by the impulse, it would be increased by increasing the rod length.

Figure 7 shows the ratios of each impulse element over the total impulse for 2, 4, 6, and 8-cm rods. Drag impulse is decreased by about $10 \%$ with $2 \mathrm{~cm}$ increments in the rod length. On the other hand, all other impulse elements increase as the rod length increases. For the $2-\mathrm{cm}$ rod, the ratio of buoyancy impulse and surface tension impulse over the total impulse is $2.3 \%$, whereas for the 8 -cm rod, it increased up to $19.6 \%$.

\section{Conclusion}

We have analyzed the relative magnitudes of four force elements (mass force, drag force, buoyancy force, force by the viscous flow, and surface tension force) according to the rod length, when hydrophobic rods have rotational motion with high speed moving into water from the water surface. In addition, the dominant impulse element generated by each force has been investigated. While added mass force and drag force are dominant regardless of the rod length, buoyancy force and surface tension force are relatively small. In the case of the rod lengths of 2, 4, $6 \mathrm{~cm}$, although drag forces were higher than added mass forces when the rotational motion starts, the magnitudes of the two forces were reversed during rotation. For these three rod lengths, it was found that reversals of magnitudes of the two forces occurred when the velocity 

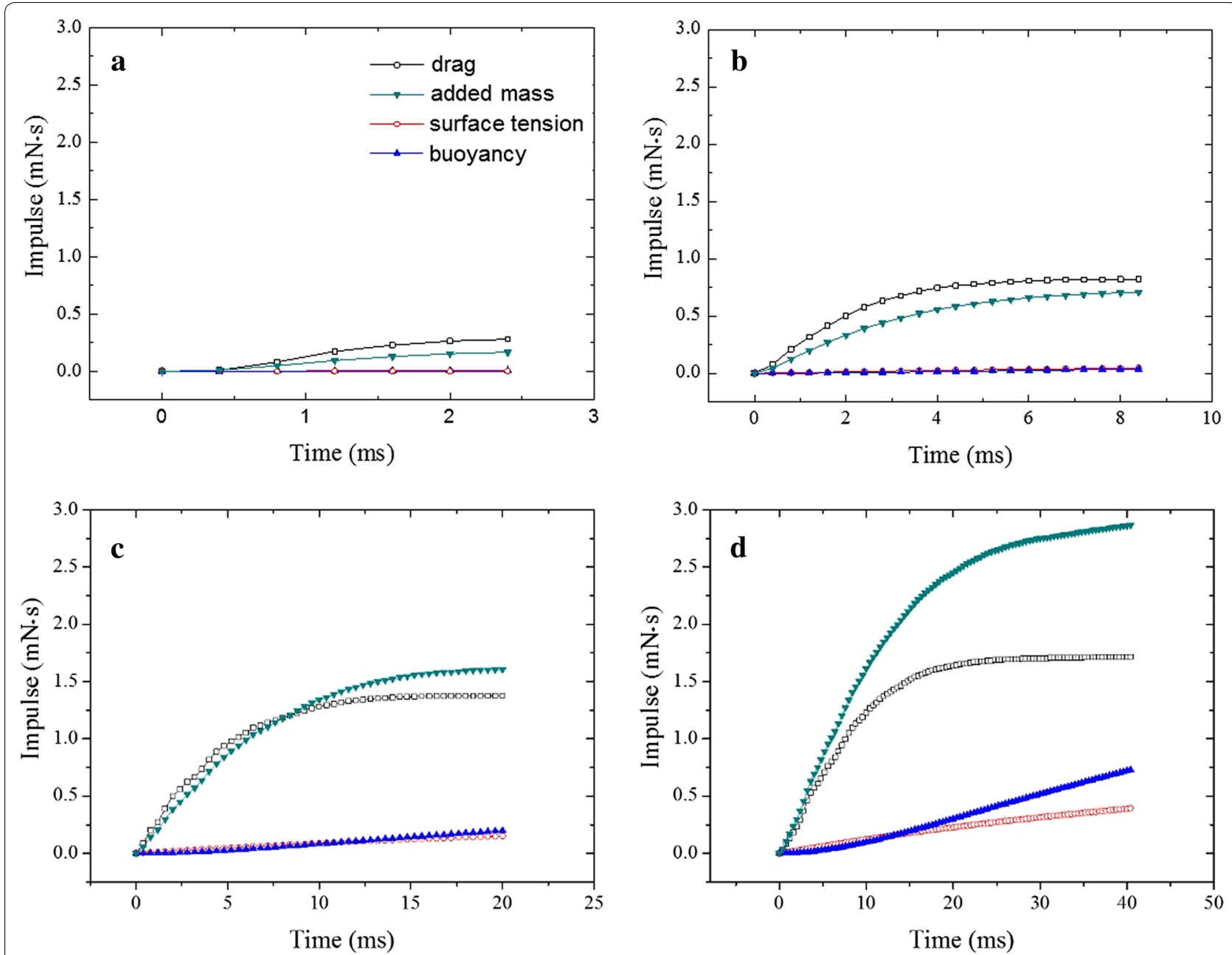

Fig. 6 The change of the magnitude of four impulse elements according to time for each rod length: $\mathbf{a} 2 \mathrm{~cm}, \mathbf{b} 4 \mathrm{~cm}, \mathbf{c} 6 \mathrm{~cm}$, and $\mathbf{d} 8 \mathrm{~cm}$

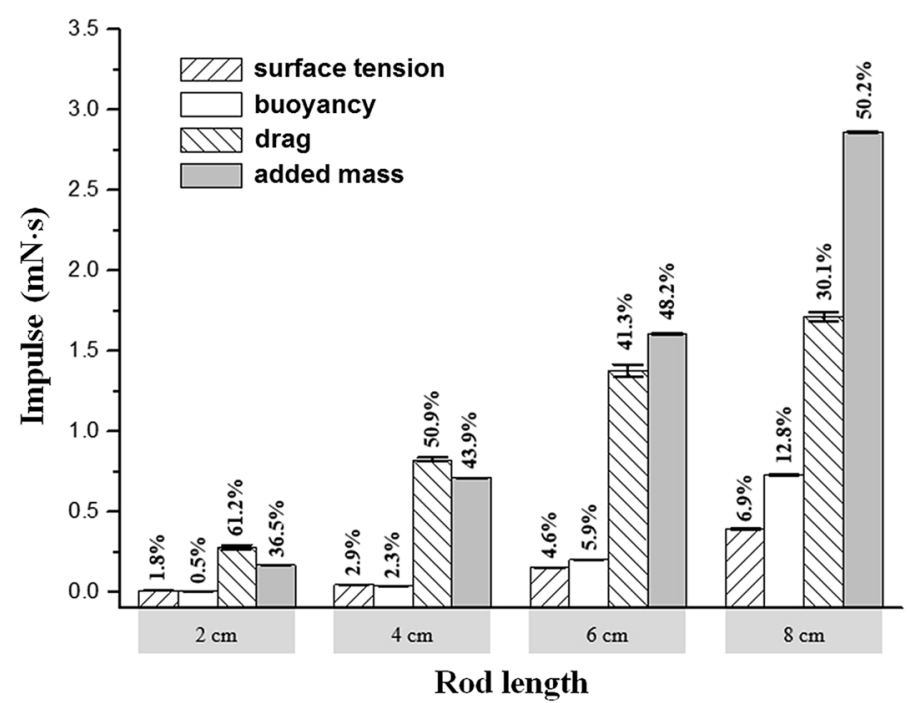

Fig. 7 The ratios of each impulse element over the total impulse of each rod length 
at the end span of the rod was $0.5 \mathrm{~m} / \mathrm{s}$. However, in the case of the rod length of $8 \mathrm{~cm}$, added mass force was higher than drag force throughout the rod motion.

Added mass impulse and drag impulse are considerably larger than those by buoyancy and surface tension impulses. Among the two impulses, while drag impulse is larger than added mass impulse for short rod lengths, magnitudes of the two impulses reversed for long rod lengths. However, buoyancy impulse and surface tension impulse gradually increased according to the increase of the rod length. The maximum momentary forces did not significantly decrease according to the increase of a rod; the duration time of the force, on the other hand, increased considerably. The increased duration time of the force is a key factor to increase the impulse for the larger rod. A high impulse contributes to increased jumping height of a jumping object.

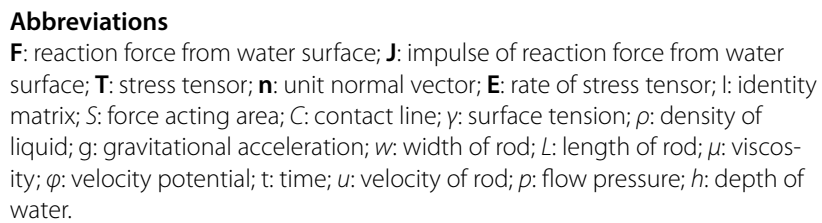

\section{Authors' contributions}

SGP, MCL, and HJT participated in design, fabrication, and test the device and drafted the manuscript. JSK conceived of the study, reviewed all test methods and results, and finalized the drafted manuscript. Both authors read and approved the final manuscript.

\section{Competing interests}

The authors declare that they have no competing interests.

\section{Funding}

This research was a part of the project titled "Development of continuous flow type multi-purpose oil recovery USV (Unmanned Surface Vehicle) using oil separation Filter", funded by the Ministry of Oceans and FisheriesKorea.

\section{Publisher's Note}

Springer Nature remains neutral with regard to jurisdictional claims in published maps and institutional affiliations.

Received: 13 July 2018 Accepted: 4 January 2019

Published online: 11 January 2019

\section{References}

1. Glasheen JW, Mcmahon TA (1996) Size-dependence of water running ability in basilisk lizard. J Exp Biol 199:2611-2618

2. Gao X, Jiang L (2004) Water-repellent legs of water striders. Nature 432:36

3. Suter RB, Gruenwald J (2000) Predator avoidance on the water surface: kinematics and efficacy of vertical jumping by dolomedes. J Aachnol 28:201-210

4. Suhr SH, Song YS, Lee SJ, Sitti M (2005) Biologically inspired miniature water strider robot. In: Proceedings of the robotics: science and systems I, Boston, USA. pp 319-325

5. Takonobu H, Kodaira K, Takeda H (2005) Water Strider's muscle arrangement-based robot. In: IEEE international conference on intelligent robots and systems (IROS), Edmonton, Canada. pp 1754-1759

6. Song YS, Suhr SH, Sitti M (2007) Modeling of the supporting legs for designing biomimetic water strider robots. In: IEEE international conference on robotics and automation (ICRA), Roma, Italy. pp 2303-2310

7. Suzuki K, Takanobu H, Noya K, Koike H, Miura H (2007) Water strider robots with microfabricated hydrophobic legs. In: IEEE international conference on intelligent robots and systems (IROS), San Diego, USA. pp 590-595

8. Lee DG, Kim HY (2008) Impact of a superhydrophobic sphere onto water. Langmuir 24:142-145

9. Shin BS, Kim HY, Cho KJ (2008) Towards a biologically inspired small-scale water jumping robot. In: Proceedings of the 2nd Biennial IEEE/RAS-EMBS international conference on biomedical robotics and biomechatronics (BIOROB 2008), Scottsdale, USA. pp 127-131

10. Matsuyama Y, Hirai S (2007), Analysis of circular robot jumping by body deformation. In: IEEE international conference on robotics and automation (ICRA), Roma, Italy. pp 1968-1973

11. Bush JWM, Hu DL (2006) Walking on water: biolocomotion at the interface. Annu Rev J Fluid Mech 38:339-369

12. Bush JWM, Hu DL, Prakash M (2008) The integument of water-walking arthropods: form and function. Adv Insect Physiol 34:117-192

13. Hu DL, Bush JWM (2010) The hydrodynamics of water-walking arthropods. J Fluid Mech 644:5-33

\section{Submit your manuscript to a SpringerOpen ${ }^{\circ}$ journal and benefit from:}

- Convenient online submission

$\checkmark$ Rigorous peer review

- Open access: articles freely available online

- High visibility within the field

- Retaining the copyright to your article

Submit your next manuscript at $\boldsymbol{\nabla}$ springeropen.com 